\title{
Calculation of Neutron EDM in quenched and full QCD
}

\author{
CP-PACS Collaboration: E. Shintani, ${ }^{1+}$ S. Aoki, ${ }^{1,2}$ N. Ishizuka,,${ }^{1,3}$ K. Kanaya, ${ }^{1}$ Y. \\ Kuramashi, ${ }^{1,3}$ M. Okawa, ${ }^{4}$ A. Ukawa, ${ }^{1,3}$ T. Yoshié, ${ }^{1,3}$. \\ ${ }^{1}$ Graduate School of Pure and Applied Sciences, University of Tsukuba \\ ${ }^{2}$ Riken BNL Research Center, Brookhaven National Laboratory \\ ${ }^{3}$ Center for Computational Sciences, University of Tsukuba \\ ${ }^{4}$ Department of Physics, Hiroshima University
}

\begin{abstract}
We report on a direct lattice calculation of the neutron $\operatorname{EDM}(\mathrm{NEDM})$ using the external electric field method in both quenched and full QCD. In quenched QCD, we use a $24^{3} \times 32$ lattice at $\beta=2.6$ with the Iwasaki gauge action and the clover fermion action to examine the viability of this method. In particular we investigate possible effects of violation of the periodic boundary condition of the external electric field on the NEDM signal. We also study the quark mass dependence of NEDM in quenched QCD, and observe that NEDM seems to remain non-zero toward the chiral limit because of the quenched artifact. In 2-flavor full QCD we employ configurations generated by the CP-PACS collaboration on a $24^{3} \times 48$ lattice at $\beta=2.1$ with the same gluon and quark actions as in the quenched case. Since the number of configurations is limited, we employ 8 different source points per one configuration and take an average over them. Our preliminary result at three quark masses $\left(m_{P S} / m_{V} \simeq 0.81,0.76,0.69\right)$ indicates that non-zero value for NEDM can be obtained in full QCD. Statistical errors, however, are still too large to show the theoretically expected behaviour for NEDM in full QCD that it vanishes in the chiral limit.
\end{abstract}

UTCCS-P-26

XXIV International Symposium on Lattice Field Theory

July 23-28 2006

Tucson Arizona, US

\footnotetext{
*E-mail:shintani@het.ph.tsukuba.ac.jp

† Speaker.
} 


\section{Introduction}

Calculating the neutron electric dipole moment (NEDM) in the presence of the $\theta$ term of QCD has been known to be a difficult task. Various effective models in fact give order of magnitude different results for NEDM. Nonetheless, combining these rough estimates with the experimental upper bound on NEDM given by $d_{N} \leq 6.3 \times 10^{-13} \mathrm{e} \cdot \mathrm{fm}$ [四] yields a bound on $\theta, \theta<10^{-10}$, which confirms the existence of the strong $\mathrm{CP}$ problem. Our aim here is to give a more accurate and reliable estimate of NEDM in the presence of the $\theta$ term based on lattice QCD calculations. Such a result will be indispensable in order to extract the value of $\theta$ from a non-zero value of NEDM should it be observed in future experiments.

The first lattice calculation of NEDM failed to obtain a signal[2]. Fifteen years later we proposed a formulation to extract NEDM form factors and numerically verified that the method works in quenched QCD with the domain-wall fermion[3]. In the same year, this formulation was employed in a full QCD calculation with the domain-wall fermion[ $₫$ ]. They, however, could obtain only an upper bound on the NEDM value. At lattice 2005, we returned to the original external electric field method proposed in Ref. [2], and reported our preliminary results for NEDM from this method with both domain-wall and clover fermions in quenched QCD[5].

In this report we carry out further numerical studies in the external electric field method with the clover fermion. We first investigate the problem with this method that a large discontinuity (gap) appears in the uniform electric field at the boundary in the time direction violating the periodic boundary condition. From this investigation we learn how we can minimize the unwanted effect of the gap to NEDM signals. Using this knowledge, we apply the method to both quenched and full QCD calculations at several quark mass parameters in order to investigate the quark mass dependence of NEDM.

\section{Formulation}

In our calculation, NEDM is extracted from the interaction term between the electric field $\vec{E}$ and the neutron spin $\vec{S}$ in the Hamiltonian:

$$
\delta H_{\mathrm{CP}}=d_{N}(\theta) \vec{S} \cdot \vec{E}
$$

where $d_{N}(\theta)$ represents the magnitude of NEDM, and $\theta$ is the CP violating phase of the strong interaction. For small $\theta$ we can expand $d_{N}(\theta)=d_{N} \theta+O\left(\theta^{3}\right)$, and hereafter we ignore higher order terms in $\theta$.

A uniform and static electric field in Minkovski space is introduced by replacing the link variable as

$$
U_{i}(x) \longrightarrow \tilde{U}_{i}(x)=U_{i}(x) e^{q_{e} E_{i} t}, \quad U_{i}^{\dagger}(x) \longrightarrow \tilde{U}_{i}^{\dagger}(x)=U_{i}^{\dagger}(x) e^{-q_{e} E_{i} t},
$$

where $q_{e}$ represents the quark charge: $q_{e}=2 / 3$ for up quark and $q_{e}=-1 / 3$ for down quark. This replacement, however, destroys the periodicity of $U_{i}(x)$ at the boundary $t=T$ :

$$
\tilde{U}_{i}(\vec{x}, t=T)=U_{i}(\vec{x}, T) e^{q_{e} E_{i} T} \neq \tilde{U}_{i}(\vec{x}, t=0)=U_{i}(\vec{x}, t=0),
$$

As a consequence, uniformity of the electric field is violated between $t=0$ and $t=T$. In section 3.1 we will numerically investigate the effect of this non-uniformity to the EDM signal. 
To include the effect of the $\theta$ term, we reweight the nucleon propagator with $e^{i \theta Q}$ :

$$
\left\langle N_{\alpha} \bar{N}_{\beta}\right\rangle_{\theta}=\left[\left\langle N \bar{N} e^{i \theta Q}\right\rangle\right]_{\alpha \beta}
$$

where $\alpha, \beta$ denotes spinor indicies of the nucleon. We may reduce the sign problem due to the complex reweighting factor $e^{i \theta Q}$ by taking $\theta$ small. On the other hand, we have to accumulate a large number of configurations to ensure correct distribution of topological charge $Q$, since results of NEDM strongly depend it.

For the electric field in $z$ direction, $E=(0,0, E)$, the ratio of nucleon propagator between spin up and down components has the following $t$ dependence:

$$
R_{3}^{\text {naive }}(E, t ; \theta)=\frac{\left\langle N_{1} \bar{N}_{1}\right\rangle_{\theta}(E, t)}{\left\langle N_{2} \bar{N}_{2}\right\rangle_{\theta}(E, t)}=Z_{N}(E, \theta) e^{-d_{N} \theta E t}+\cdots
$$

where $Z_{N}(E, \theta)$ is constant in $t$ but depends on $E$ and $\theta$, and the dots represent contributions from excited states. We further take the following triple ratio in order to subtract fake contributions from $E=0$ and $\theta=0$ caused by finite statistics.

$$
R_{3}(E, t ; \theta)=\frac{R_{3}^{\text {naive }}(E, t ; \theta)}{R_{3}^{\text {naive }}(0, t ; \theta)} \times \frac{R_{3}^{\text {naive }}(0, t ; \theta=0)}{R_{3}^{\text {naive }}(E, t ; \theta=0)} .
$$

We finally take the ratio between $E$ and $-E$, in order to remove $O\left(E^{2}\right)$ contributions as

$$
R_{3}^{\text {corr }}(E, t ; \theta)=\frac{R_{3}(E, t ; \theta)}{R_{3}(-E, t ; \theta)}=\frac{R_{3}^{\text {naive }}(E, t ; \theta)}{R_{3}^{\text {naive }}(-E, t ; \theta)} \frac{R_{3}^{\text {naive }}(E, t ; \theta=0)}{R_{3}^{\text {naive }}(-E, t ; \theta=0)}=Z_{N} e^{-2 d_{N} \theta E t}+\cdots .
$$

In section 3 we will show the time dependence of $R_{3}(E, t ; \theta)$ for both $E$ and $-E$, and we will also plot the effective mass of $R_{3}^{\text {corr }}$.

\section{Numerical results}

In our calculation we employ the RG improved (Iwasaki) gauge action and the clover fermion action with the tadpole improved tree value of $c_{s w}$. The $O\left(a^{2}\right)$ improved field theoretical definition of the topological charge is employed, after 20 cooling steps in quenched QCD and 50 cooling steps in full QCD. In Fig. 1 the histogram of the topological charge distribution is plotted in quenched QCD at $\beta=2.6$ (leftmost) and in full QCD at $\beta=2.1$ and $K_{\text {sea }}=0.1357$ (second from left), $K_{\text {sea }}=0.1367$ (third), $K_{\text {sea }}=0.1374$ (fourth). Smeared sources with the form of $A e^{-B r}$ is employed in our calculation. In Fig. 2, the effective mass of nucleon at $E=0$ and $\theta=0$ is plotted in quenched QCD (left) and full QCD (right) at several values of the quark mass.

\subsection{Effects of non-uniform electric field in quenched QCD}

We first investigate the effect of a non-uniform electric field at the boundary to EDM signals, using quenched QCD on a $24^{3} \times 32$ lattice at $\beta=2.6$, which corresponds to $a^{-1} \simeq 2 \mathrm{GeV}$. We use $E=0.004$ and $\theta=0.1$ in this study. We always put the gap (discontinuity) of the electric field between $t=T$ and $t=1$, denoting this as $t_{E}=1$. For comparison, we place the nucleon source at two different time slices: $t_{\mathrm{src}}=1$ and $t_{\mathrm{src}}=8$. To reduce the computational cost we take a heavy 

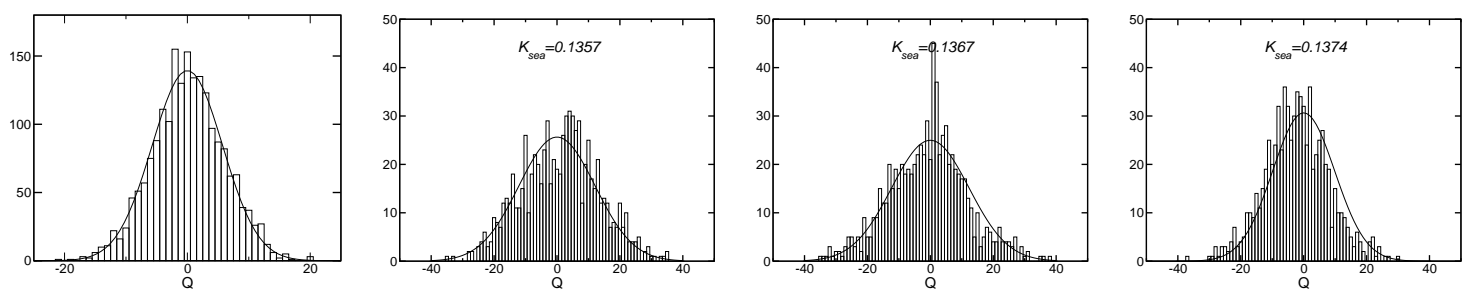

Figure 1: Histogram of topological charge distribution in quenched QCD at $\beta=2.6$ (first) and full QCD at $\beta=2.1$ with $K_{\text {sea }}=0.1357$ (second), $K_{\text {sea }}=0.1367$ (third), $K_{\text {sea }}=0.1374$ (fourth).
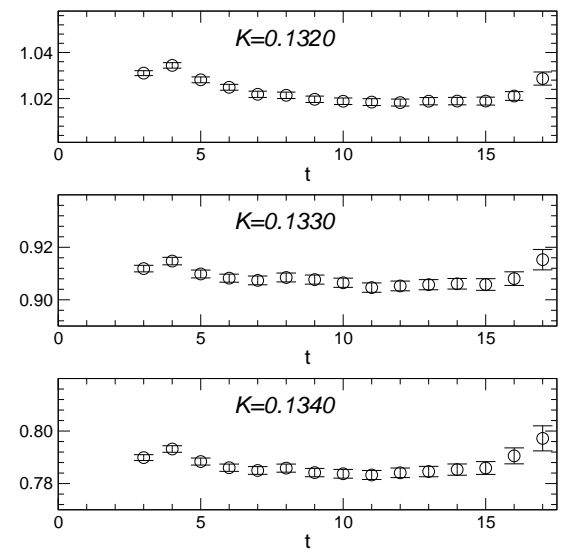

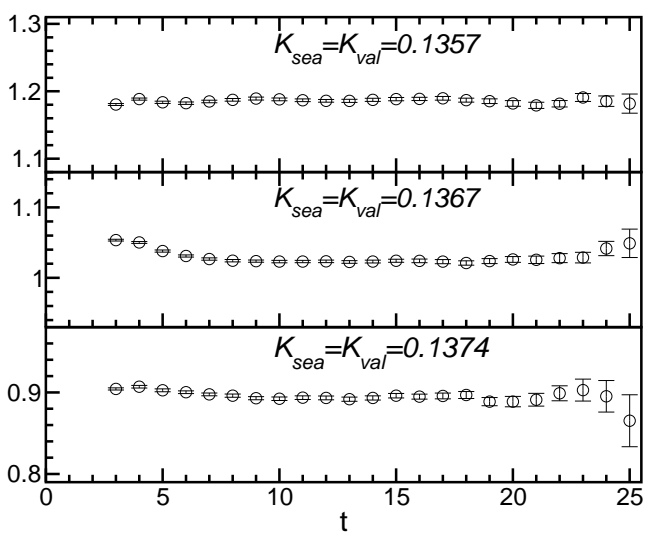

Figure 2: Effective mass plot of nucleons in quenched QCD(left) and full QCD(right).

fermion mass $K=0.1320$ corresponding to $m_{P S} / m_{V} \simeq 0.85$. On the left of Fig. B we compare the time dependence of $R_{3}(E, t ; \theta)$ between $t_{\mathrm{src}}=1$ and $t_{\mathrm{src}}=8$. We observe that the $t$ dependences are much different between the two cases near source points $\left(t-t_{\mathrm{src}}+1 \leq 5\right)$. On the other hand, at large $t\left(t-t_{\mathrm{src}}+1 \geq 8\right)$, the two cases show similar behaviors. This indicates that the gap affects the EDM signal near $t=t_{E}$ while its influence almost disappears at large $t$ which satisfies $t-t_{E} \geq 8$. Therefore the result with $t_{\mathrm{src}}=8$ is expected to be free from the influence of the gap.

This tendency is also seen from the comparison of the effective mass plot between two sources, given on the right of Fig. 3. We observe a plateau at $6 \leq t-t_{\mathrm{src}}+1 \leq 10$ for the case of $t_{\mathrm{src}}=8$. For the case of $t_{\mathrm{src}}=1$, there is a candidate for a plateau at $6 \leq t-t_{\mathrm{src}}+1 \leq 10$. However, the value in this range of $t$ disagrees with that from the plateau of $t_{\mathrm{src}}=8$ data. Therefore, we should conclude that this is not a real plateau, but a fake effect due to the gap. In fact, looking further away from the source, we observe a plateau at the range $8 \leq t-t_{\mathrm{src}}+1 \leq 12$ for $t_{\mathrm{src}}=1$ whose value is in agreement with that for $t_{\text {src }}=8$.

Our study concludes that we should place the source point as far from the gap as possible in order to avoid the influence of the gap to EDM signals. The condition that $t_{\mathrm{src}}-t_{E} \geq 8$ seems necessary in this method for the EDM calculation.

\subsection{Preliminary results in full QCD}

We apply our method of EDM calculations to $N_{f}=2$ flavor full QCD configurations on a $24^{3} \times 48$ lattice at $\beta=2.1$ corresponding to $a^{-1} \simeq 2 \mathrm{GeV}$, generated by CP-PACS collaboration[6] using RG improved gauge and tadpole improved clover fermion actions. In this calculation we take $E=0.004$ and $\theta=0.025$ and employ four values for valence quark masses, which are equal to sea 

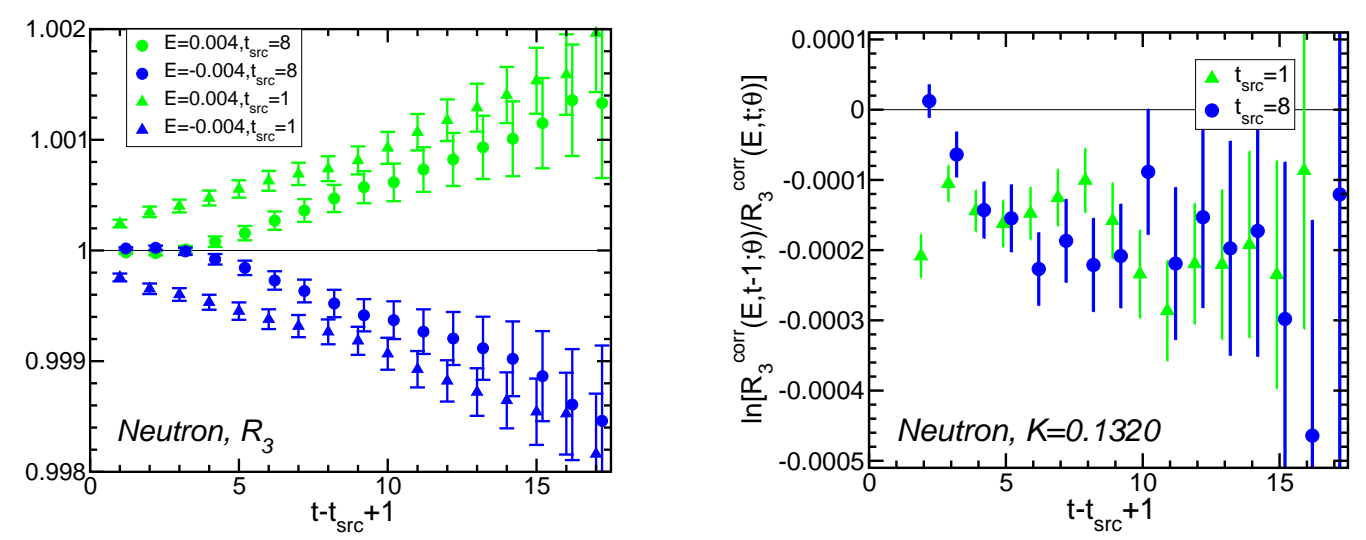

Figure 3: Time dependence of $R_{3}(E, t ; \theta)$ (left) and effective mass (right) for $t_{\mathrm{src}}=1$ and $t_{\mathrm{src}}=8$ in quenched QCD.

quark masses of full QCD ( $\left.K_{\text {sea }}=0.1357,0.1367,0.1374,0.1382\right)$ in order to perform the chiral extrapolation.

As shown in section 3.1, the influence of the non-uniform electric field at the boundary to the EDM signal is largely suppressed if $t_{\mathrm{src}}-t_{E} \geq 8$. In this full QCD calculation, we take the maximal separation, namely $t_{\mathrm{src}}-t_{E}=24$ on a $24^{3} \times 48$ lattice. Since the distance between the gap and the source for the positive time direction $\left(t_{\mathrm{src}}-t_{E}=24\right)$ is same as the one for the negative time direction $\left(48+t_{E}-t_{\mathrm{src}}=24\right)$ in this setup due to the periodic boundary condition in time, we can utilize both forward and backward propagations of nucleon for EDM calculations.

Our study in quenched QCD indicates that the number of full QCD configurations available (about 770) is not sufficient for our method of EDM calculations. In order to effectively increase statistics, we employ 8 different source points per one configuration, choosing $\left(t_{\mathrm{sec}}, t_{E}\right)=(6,30)$, $(12,36),(18,42),(24,48),(30,6),(36,12),(42,18),(48,24)$. The decrease of statistical errors we observe in our results indicates that data from these source points are almost independent. Therefore the total number of statistics is more than 12,000 , including forward and backward propagations.

The top panels of Fig. $\theta$ plot $R_{3}(E, t ; \theta)$ as a function of $t$ for $E$ and $-E$ for each $K_{\text {val }}$. We clearly observe non-zero signals, which shows the expected dependence on the sign of $E$. Furthermore the sign of the slope (positive for positive $E$ ) agrees with that in the quenched case. By fitting $R_{3}^{\text {corr }}$ with the exponential form 2.7 over $6 \leq t-t_{\mathrm{src}}+1 \leq 10$ we obtain

$$
d_{N}=-0.028(17) \mathrm{e} \cdot \mathrm{fm},
$$

at $K=0.1357$. Similarly we have

$$
d_{N}=-0.033(22) \mathrm{e} \cdot \mathrm{fm}, \quad d_{N}=-0.032(25) \mathrm{e} \cdot \mathrm{fm},
$$

at $K=0.1367$ and $K=0.1374$. The statistical error is still large.

\subsection{Quark mass dependence in quenched and full QCD}

As is well known, NEDM vanishes in the zero quark mass limit since $\theta$ term can be transformed to $m \bar{\psi} e^{i \gamma_{5} \theta} \psi$ by a chiral rotation. In quenched QCD, however, this property does not hold since the quark determinant is set to unity. There exist no QCD-based theoretical prediction for 

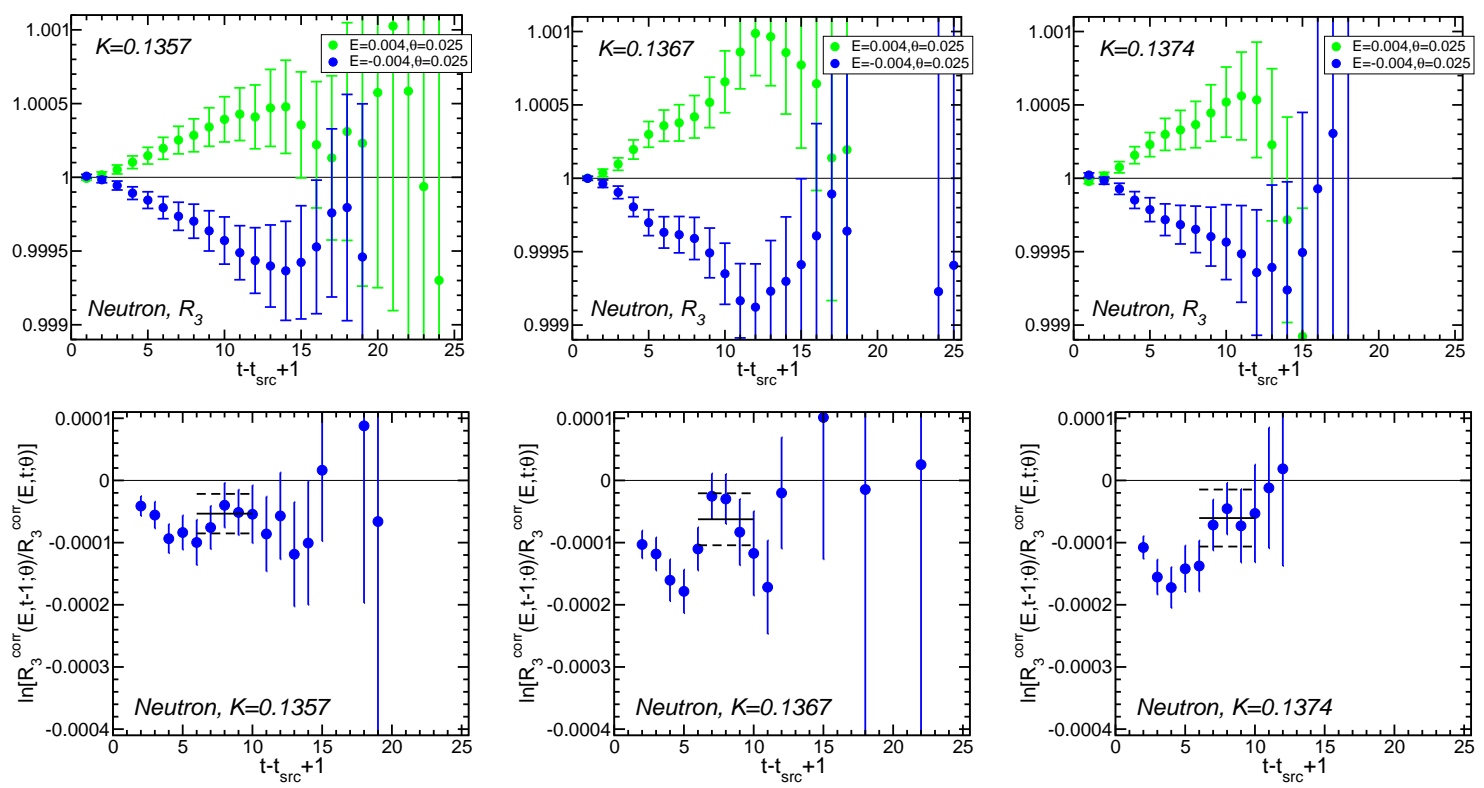

Figure 4: Time dependence of $R_{3}$ function(top) and effective mass plot of $R_{3}^{\text {corr }}$ (bottom) at $K=K_{\text {sea }}=K_{\text {val }}=$ $0.1357,0.1367,0.1374$ averaged over 8 source sets. The straight line in the right figure is the result from the global fit.

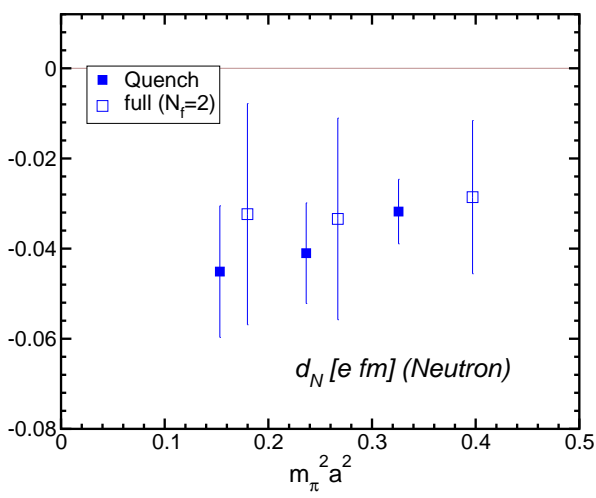

Figure 5: NEDM as a function of pion mass squared in quenched and full QCD.

the quark mass dependence of NEDM in quenched QCD. The instanton liquid model [7] predicts a $1 / m_{q}^{2} \sim 1 / m_{\pi}^{4}$ divergence, while partially quenched chiral perturbation theory[8] leads to the $1 / m_{\pi}^{3}$ behavior toward the chiral limit.

In Fig. 5 we plot values of NEDM as a function of pion mass squared. In quenched QCD these values are obtained after averaging over three direction of the electric field. We take $8 \leq$ $t-t_{\mathrm{src}}+1 \leq 12$ as the fitting range since $t_{\mathrm{src}}=t_{E}=1$ is employed in this quenched calculation. We clearly observe a non-vanishing behavior toward the chiral limit in quenched QCD. We are not able to determine, however, the precise quark mass dependence to distinguish $1 / m_{\pi}^{4}$ from $1 / m_{\pi}^{3}$ due to large statistical errors and lack of sufficient quark mass points. In full QCD statistical errors are too large to confirm that NEDM vanishes in the chiral limit. We are currently increasing the number of source points to reduce statistical errors. 


\section{Conclusions}

In this report we presented our lattice calculation of NEDM using the external electric field method. In quenched QCD we investigate the effect from the gap (discontinuity) of the electric field, by comparing different two source points at the fixed gap point. From this investigation we learn that the effect of the gap to NEDM signals can be reduced by placing the source point away from the gap. We also obtained results which show that NDEM does not vanish toward the chiral limit, as indicated by some models. This, however, is a quenching artifact.

In two-flavor full QCD, we obtained, for the first time in full QCD simulations, a non-zero value for NEDM. This is made possible by carefully avoiding the effect of the gap in the electric field by placing the source as far away as possible from the gap, and by accumulating a large number of statistics (over 12,000) by averaging over 8 source points and over both forward and backward propagation of the nucleon. The statistical error is still large (60-80\%). Clearly we have to increase statistics in our full QCD calculations, by employing more source points and/or different directions of the electric field, in order to perform a reliable chiral extrapolation of NEDM to the physical quark mass. After this attempt, we will proceed to our final project, a calculation of NEDM in $N_{f}=2+1$ full QCD.

This work is supported in part by Grant-in-Aid of ministry of Education (Nos. 13135204, 13135216, 15540251, 16540228, 17340066, 17540259, 18104005, 18540250 ) Numerical simulations are performed on Hitachi SR11000 at High Energy Accelerator Research Organization (KEK), Hiroshima University and Tokyo University. At KEK this simulation is under support of Large Scale Simulation Program (No. 06-04).

\section{References}

[1] P. G. Harris et al., Phys. Rev. Lett. 82, 904 (1999).

[2] S. Aoki, A. Gocksch, Phys. Rev. Lett. 63, 1125 (1989); erratum, ibid. 65, 1172 (1990).

[3] E. Shintani, et al., Phys. Rev. D72, 014504 (2005).

[4] F. Berruto, T. Blum, K. Orginos, A. Soni, Nucl. Phys. Proc. Suppl. 140, 411 (2005); T. Blum, Proc. Sci. LAT2005, 010 (2005); F. Berruto, T. Blum, K. Orginos, A. Soni, Phys. Rev. D73, 054509 (2006).

[5] E. Shintani et al., talk given at LATTICE'05, hep-lat/0509123

[6] S. Aoki, et al., (CP-PACS Collaboration), Phys. Rev. D65, 054505 (2002); erratum ibid. D67, 059901 (2003).

[7] P. Faccioli, D. Guadagnoli, S. Simula, Phys. Rev. D70, 074017 (2004).

[8] D. O'Connell and M. J. Savage, Phys. Lett. B633, 319 (2006). 